\title{
Minimally invasive video-assisted thyroidectomy: seven-year experience with 240 cases
}

\author{
Marcin Barczyński, Aleksander Konturek, Małgorzata Stopa, Aleksandra Papier, Wojciech Nowak \\ Third Department of General Surgery, Jagiellonian University Medical College, Krakow, Poland
}

Videosurgery Miniinv 2012; 7 (3): 175-180 DOI: $10.5114 /$ wiitm.2011.28871

\begin{abstract}
Introduction: Minimally invasive video-assisted thyroidectomy (MIVAT) has gained acceptance in recent years as an alternative to conventional thyroid surgery.

Aim: Assessment of our 7-year experience with MIVAT.

Material and methods: A retrospective study of 240 consecutive patients who underwent MIVAT at our institution between 01/2004 and 05/2011 was conducted. The inclusion criterion was a single thyroid nodule below $30 \mathrm{~mm}$ in diameter within the thyroid of $25 \mathrm{ml}$ or less in volume. The exclusion criteria were previous thyroid or parathyroid surgery, $T 2$ or higher thyroid cancer, N1 stage, and thyroiditis. The Miccoli technique was used. The analysis included indications, eligibility rate, operative time, morbidity and cosmetic effects.

Results: Of 6,574 patients referred for thyroid surgery, 240 (3.6\%) were eligible for MIVAT. In the final pathology report, there were 206 follicular adenomas, 21 papillary thyroid cancers, 9 cases of Graves' disease and 4 follicular cancers. Reasons for exclusion were as follows: thyroid volume above $25 \mathrm{ml}$ in 5401 (85.3\%), thyroid cancer larger than stage $T 1$ in 392 (6.2\%), thyroiditis in 358 (5.6\%), and previous neck surgery in 183 patients (2.9\%). Minimally invasive videoassisted thyroidectomy operations consisted of 210 lobectomies and 30 total thyroidectomies, including 15 one-stage parathyroidectomies. Mean operative time was $38.6 \pm 15.1 \mathrm{~min}$. Transient versus permanent recurrent laryngeal nerve injury was found in 8 (3.0\%) vs. $2(0.7 \%)$ nerves at risk, respectively. Cosmetic effects were assessed after 1 and 6 months of follow-up as very good or excellent by $89.6 \%$ and $95.8 \%$ of patients, respectively.

Conclusions: Minimally invasive video-assisted thyroidectomy is suitable for surgeons experienced in thyroid and video-assisted surgery. It is feasible for well-selected patients including cases of T1 thyroid cancer, Graves' disease and concomitant parathyroid adenoma.
\end{abstract}

Key words: minimally invasive surgery, video-assisted thyroidectomy, benign thyroid disease, thyroid cancer.

\section{Introduction}

Thyroidectomy is one of the most common operations performed in endocrine surgery. The basic principles of safe and efficient thyroid surgery established in the early $20^{\text {th }}$ century have remained unchanged, with only a few minor refinements to the technique [1].

Multiple endoscopic approaches for thyroid excision have been developed, ranging from a gasless, video-assisted central or lateral approach to a complete endoscopic supraclavicular, axillary, anterior chest wall or breast approach and even robotic surgery [2]. Among all these new operations, minimally invasive video-assisted thyroidectomy (MIVAT) described in 1998 by Miccoli has gained increasing popularity in Europe over the last 15 years [3]. It is safe and offers improved outcomes of surgery in terms of

Address for correspondence:

Marcin Barczyński MD, PhD, FEBS-ES, Third Department of General Surgery, Jagiellonian University Medical College, 37 Prądnicka,

31-202 Krakow, Poland, phone: +48 6023759 39, fax: +48 1263331 05, e-mail: marbar@mp.pl 
decreased postoperative pain, a shorter scar and greater cosmetic satisfaction when compared to conventional thyroidectomy [4]. In experienced hands, it is suitable not only for removal of small and benign unilateral thyroid nodules, but also for more demanding operations, such as total thyroidectomy for Graves' disease or low-risk thyroid cancer $[5,6]$.

\section{Aim}

The aim of this study was to evaluate our 7-year experience with MIVAT.

\section{Material and methods}

This is a retrospective study of 240 consecutive patients who underwent MIVAT between January 2004 and May 2011 and were prospectively registered at our institutional computer-based register of thyroid surgery. The study protocol was approved by the Institutional Review Board. The eligibility criteria for MIVAT were: a solitary thyroid nodule below $30 \mathrm{~mm}$ in maximum diameter, absence of both echographic and biochemical signs of thyroiditis, thyroid volume below $25 \mathrm{ml}$, no previous neck surgery or neck irradiation, and in case of suspicion of thyroid malignancy, cT1 stage according to the TNM 2010 classification.

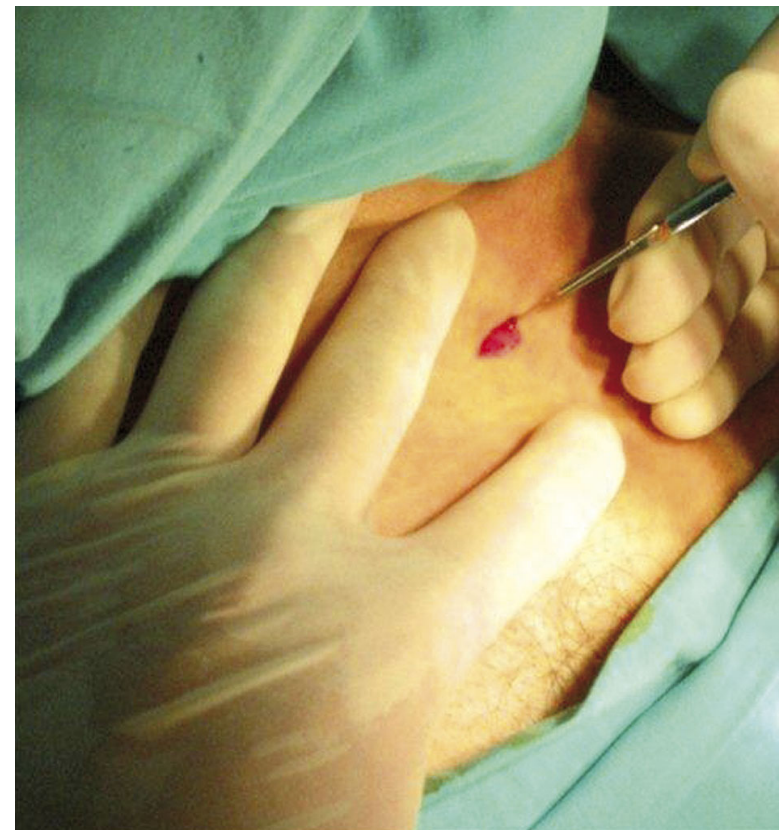

Figure 1. Skin incision for MIVAT is $2 \mathrm{~cm}$ long and is placed two fingers above the sternal notch

\section{Study endpoints and follow-up data}

The primary endpoints were indications and eligibility rate for MIVAT. The secondary endpoints were operative time, morbidity and cosmetic effects. Eligibility rate was calculated as the percentage of patients who underwent MIVAT from a cohort of all patients who underwent thyroid surgery at our institution during the study period. Operative time was calculated from skin incision to skin closure. Morbidity assessment involved prevalence of recurrent laryngeal nerve (RLN) injury, postoperative hypocalcemia, infection and bleeding. Indirect laryngoscopy was mandatory on postoperative day 1 and in case of identified early vocal cord dysfunction, during 6-month follow-up. Recurrent laryngeal nerve injury was calculated for nerves at risk and it was considered transient if unilateral vocal fold mobility was restored within 6 months following surgery, whereas it was considered permanent in cases of dysfunction lasting for at least 6 months. Postoperative hypocalcemia was diagnosed in a patient who developed a serum calcium level below $2 \mathrm{mmol} / \mathrm{l}$. Cosmetic effects were assessed on a visual analogue scale (VAS) at 1 and 6 months following the operation.

\section{Surgical technique}

All the operations consisted of MIVAT thyroid lobectomy, either unilateral or bilateral, and were performed by the same experienced surgeons. The MIVAT procedure was performed according to the Miccoli technique [3-7], with a $1.5-2 \mathrm{~cm}$ horizontal skin incision two fingers above the sternal notch (Figure 1). Having dissected but not divided the strap muscles in the midline, a working space was created by blunt dissection with a tiny spatula and retraction of the strap muscles laterally to mobilize the thyroid lobe. The gasless video-assisted approach employing a $5 \mathrm{~mm} 30^{\circ}$ rigid endoscope and other instruments designed for parathyroid and thyroid surgery (KarlStorz, Tutlingen, Germany) were used (Figure 2). The optical magnification allowed for excellent visualization of the external branch of the superior laryngeal nerve and the recurrent laryngeal nerve, which were dissected together with the upper parathyroid gland (Figure 3). The vessels of the upper thyroid pole were double-clipped and cut or were dissected and divided with the harmonic shears until the lobe was completely freed and could be extracted by gentle pulling it out through the skin incision (Figure 4). The isth- 


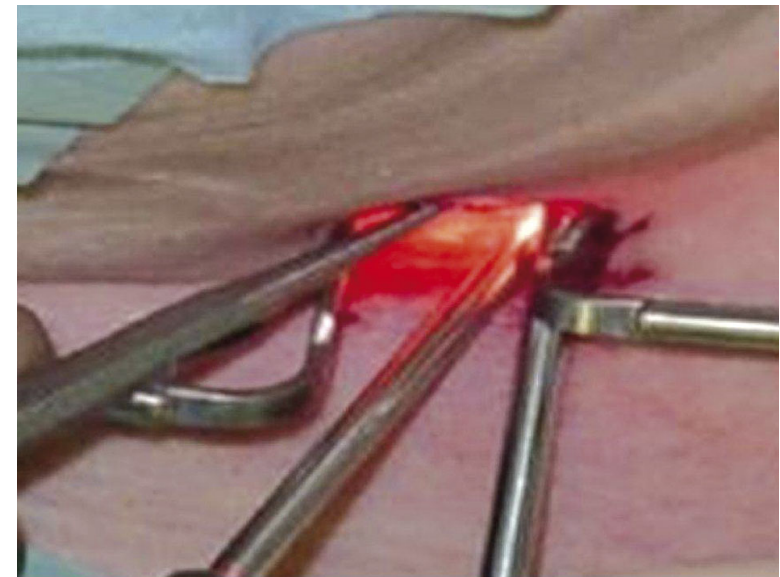

Figure 2. Working space is created by blunt dissection and retraction with hooks without gas insufflation. A $5 \mathrm{~mm}$ 30-degree oblique scope is inserted through a single neck incision together with other working instruments

mus was then dissected and divided, followed by dissection and division of the inferior thyroid pole vessels using the harmonic scalpel or a $5 \mathrm{~mm}$ clip applier and bipolar coagulation. After the final check of the recurrent laryngeal nerve, the lobe was removed and sent for histopathology. For total thyroidectomy the same technique was used for contralateral thyroid lobe dissection. The strap muscles were approximated using one stitch and the skin incision was closed with a continuous non-absorbable monofilament suture, removed routinely on the second postoperative day and replaced by Steri-strips adhesive tapes. No drainage was necessary.

\section{Statistical analysis}

The statistical significance of categorical variables was evaluated by the $\chi^{2}$-test, whereas the unpaired Student $t$ test was used for the evaluation of continuous variables (Statistica, StatSoft, Katowice). All the data were entered into a dedicated spreadsheet (Microsoft Excel 2007; Microsoft Corporation, San Jose, CA) by a medical assistant and then analyzed by a statistician. Value of $p<0.05$ was considered to indicate significance.

\section{Results}

Of 6,574 patients referred for thyroid surgery, 240 (3.6\%) were eligible for MIVAT (clinical data are summarized in Table I). The MIVAT lobectomy was car-

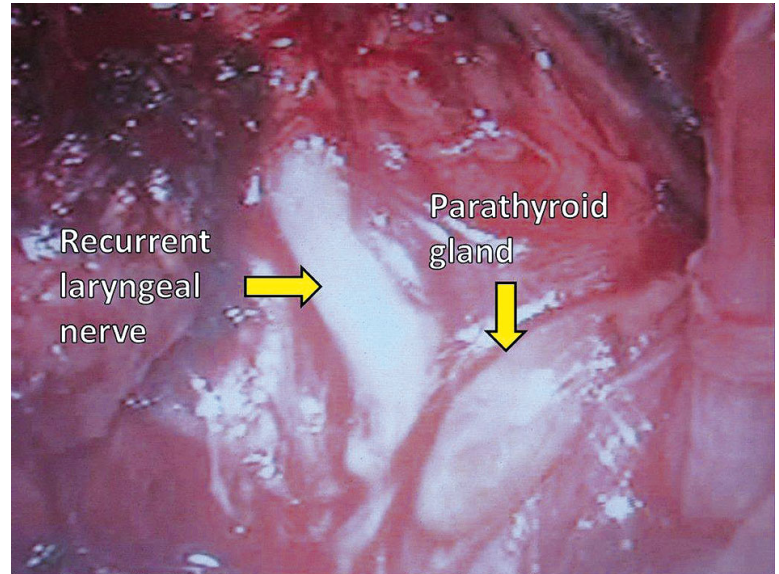

Figure 3. Recurrent laryngeal nerve and parathyroid glands should be identified before any thyroid vessel ligation. This goal is facilitated by magnification of the scope used in this videoassisted approach

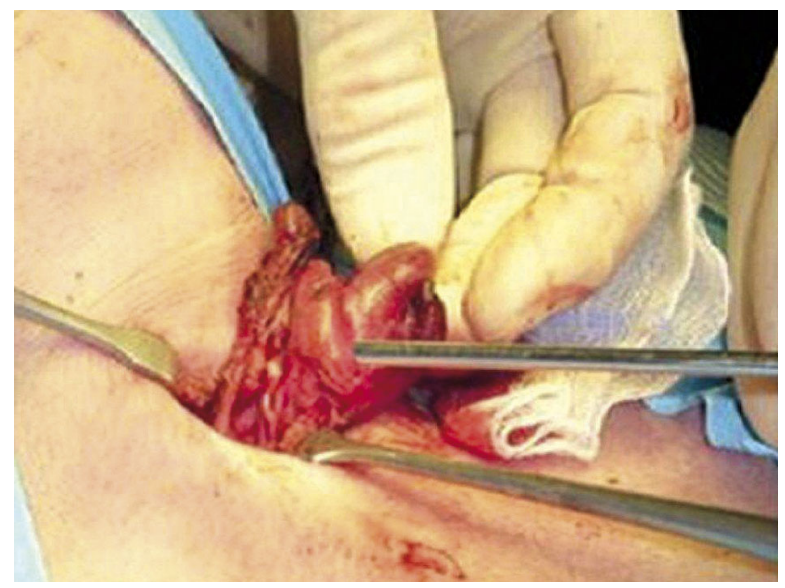

Figure 4. Having secured the superior thyroid vessels using the video-assisted approach, the thyroid lobe is gently extracted above the wound level and thyroid lobectomy is completed using open dissection

ried out in 210 patients, whereas MIVAT total thyroidectomy was performed in 30 patients, including 15 operations involving one-stage video-assisted parathyroid adenoma removal. Mean operative time of lobectomy was $33.2 \mathrm{~min}$ (range: 25-90 min), whereas for total thyroidectomy it was 45.4 min (range: 35-120 $\min )(p<0.001)$. Conversion to conventional thyroidectomy was not necessary in any of the patients in this study. In the final pathology report, there were 206 follicular adenomas, 21 papillary thyroid cancers, 9 cases 
of Graves' disease and 4 follicular cancers. These four follicular cancers were incidentally diagnosed following thyroid lobectomy and the affected patients underwent MIVAT completion thyroidectomy at 2-3 months

Table I. Clinical characteristics of 240 patients who underwent MIVAT in this study

\begin{tabular}{|c|c|}
\hline Characteristics & $\operatorname{MIVAT}(n=240)$ \\
\hline \multicolumn{2}{|l|}{ Gender, $n(\%)$} \\
\hline Male & $228(95.0)$ \\
\hline Female & $12(5.0)$ \\
\hline Mean age (range) [years] & $40.4(18-63)$ \\
\hline Mean thyroid volume (range) [ml] & $18.5(12-25)$ \\
\hline \multicolumn{2}{|l|}{ MIVAT, $n(\%)$} \\
\hline Unilateral lobectomy & $210(87.5)$ \\
\hline Total thyroidectomy & $30(12.5)$ \\
\hline \multicolumn{2}{|l|}{ Final pathology, $n(\%)$} \\
\hline Follicular adenoma & $206(85.8)$ \\
\hline Papillary cancer & $21(8.8)$ \\
\hline Graves' disease & $9(3.8)$ \\
\hline Follicular cancer & $4(1.7)$ \\
\hline \multicolumn{2}{|l|}{ Mean operative time (range) [min] } \\
\hline Unilateral lobectomy & $33.2(25-90)$ \\
\hline Total thyroidectomy & $45.4(35-120)$ \\
\hline
\end{tabular}

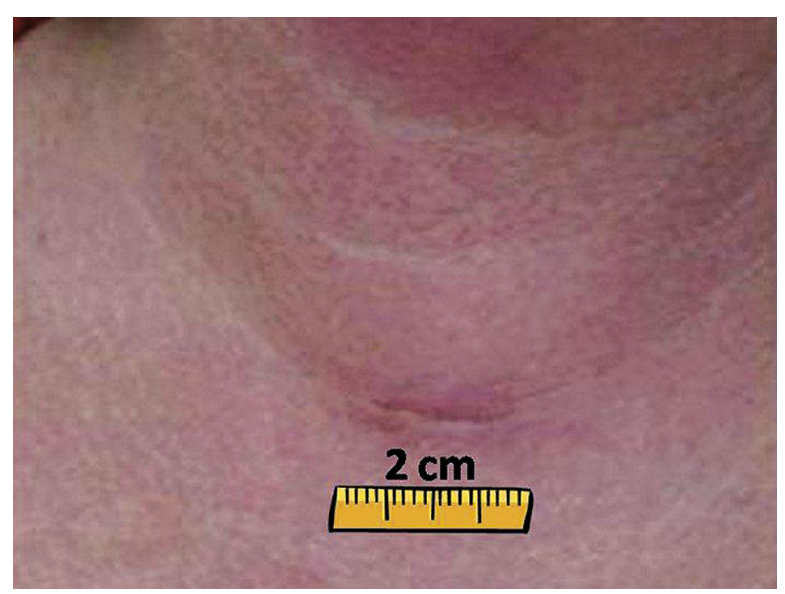

Figure 5. Four weeks after the operation, the scar following MIVAT is hardly visible, as it is hidden within one of the skin creases after the initial surgery. The reasons for exclusion from MIVAT were as follows: thyroid volume above $25 \mathrm{ml}$ in 5401 (85.3\%), thyroid cancer larger than stage T1 in 392 (6.2\%), thyroiditis in 358 (5.6\%), and previous neck surgery in 183 patients (2.9\%).

Transient versus permanent recurrent laryngeal nerve injury was found in $8(3.0 \%)$ vs. $2(0.7 \%)$ nerves at risk, respectively. Postoperative hypocalcemia was diagnosed in $2(1.0 \%)$ vs. 11 (36.7\%) patients following thyroid lobectomy versus total thyroidectomy, respectively $(p<0.001)$. Cosmetic effects were assessed after 1 and 6 months of follow-up as very good or excellent by 215 (89.6\%) and 230 (95.8\%) patients (Figure 5$)$, respectively $(p<0.001)$. Postoperative bleeding requiring reoperation was not noted in this study. Wound infection occurred in 2 cases (0.4\%) after MIVAT (unilateral lobectomy).

\section{Discussion}

Minimally invasive video-assisted thyroidectomy has become the most widely employed technique for minimally invasive thyroidectomy. The rationale behind its popularity is a standardized technique, the use of a similar approach to open thyroidectomy via a central anterior skin incision, and reproducibility of the surgical outcomes of this operation in various hands $[8,9]$.

The most common mistake of the minimally invasive surgical access to the thyroid is incorrect selection of patients. Our analysis has shown that using restrictive eligibility criteria, the proportion of patients who could be offered MIVAT was $3.6 \%$ of all patients referred for thyroid surgery at our institution, but all of them underwent this intervention successfully and we experienced no need for conversion to open thyroidectomy in this series. Nevertheless, the eligibility rate for MIVAT has been reported to range from $10 \%$ in Italy, which is recognized as an endemic goiter area, to more than $15 \%$ in non-endemic areas. Minimally invasive video-assisted thyroidectomy is particularly attractive to young female patients who need thyroid surgery and pay special attention to cosmetic effects of surgery $[7,10]$.

Some technical aspects of the MIVAT procedure are crucial for achieving safe outcomes. The magnification of the endoscope allows for easier identification of some vital structures within the neck, including the RLN and parathyroid glands. Nevertheless, gentle and complete dissection of the RLN with a spa- 
tula is recommended at the video-assisted stage of the procedure to minimize the risk of stretch injury to the RLN at the second stage of the operation, when the thyroid lobe is extracted above the wound level, which follows the division of the superior thyroid vessels [11]. The use of any energy-based device (such as harmonic shears) for hemostasis during MIVAT is safe and facilitates dissection, allowing for a significant decrease in the operative time $[12,13]$. The operation is safer when it is performed in a dry operative field, in which all the anatomical structures are clearly visible with video-assisted magnification. Nevertheless, one should be aware of the possible heat injury to the RLN and parathyroid glands, and the use of an energy-based device should be avoided in close proximity to these structures [13]. In our series of MIVAT, the prevalence of both RLN injury and hypocalcemia was similar to the results reported by others [6, 14-16]. However, it should be stressed that total thyroidectomy involves a significantly higher risk of postoperative hypocalcemia than unilateral thyroid lobectomy and the use of a videoassisted approach does not seem to reduce the risk of this adverse event.

Radford et al. in a recently published systematic review of the literature and meta-analysis aiming to compare MIVAT to conventional thyroidectomy included five trials comprising a total of 318 patients. This study demonstrated that MIVAT was as safe as the existing gold standard open thyroidectomy, but it had better cosmetic and pain outcomes for patients when compared to conventional surgery [16].

Miccoli et al. performed a systematic evidencebased literature review and analyzed use of MIVAT for benign thyroid disease [6]. This review indicated that MIVAT can be considered an appropriate treatment of some thyroid diseases, as it represents a safe procedure with the same prevalence of complications as traditional surgery, and also has advantages in terms of both cosmetic result and postoperative distress [17-19]. On the one hand, in spite of an increasing trend toward performing more extensive procedures other than thyroidectomy alone during the MIVAT operation, the current literature seems to reaffirm that the main and safest indication for MIVAT is benign disease [6]. On the other hand, since the first report of MIVAT published in 1999, the indications for this operation have been progressively extended by the Miccoli group from cytologically undetermined thyroid nodules to intermediate-risk differentiated thyroid cancers. In a recently published analysis of 1946 patients who underwent MIVAT in Pisa between 1998 and 2009, total thyroidectomy was performed in 1435 patients $(72.3 \%)$ and lobectomy in 511 cases (26.3\%) with the final histology classified as benign disease in 979 cases (51.5\%) and a malignancy diagnosed in 915 cases (48.5\%). It is of interest that unexpected thyroiditis (considered as a classic contraindication for MIVAT) was found on final histology in $17.9 \%$ of patients with benign disease and $30.9 \%$ of patients with malignancy $[7,10]$. These data confirmed the validity of the traditional indications for MIVAT (low-risk differentiated thyroid cancer, cytologically undetermined nodules and small-volume benign thyroid disease), but also indicated that the indications may be further and safely extended to patients with associated thyroiditis or with intermediate-risk differentiated thyroid cancer [7]. Minuto et al. concluded that MIVAT can be now proposed on a much larger scale than it was at its onset and cannot be considered an option for only a limited number of patients [10]. Despite these promising observations made in a tertiary referral center, it should be stressed that reproduction of these excellent outcomes should be preceded by achieving expertise in application of the technique of unilateral MIVAT lobectomy.

Our data confirm that MIVAT is suitable for surgeons experienced in thyroid and video-assisted surgery. In our hands, MIVAT is feasible for well-selected patients, including cases of T1 thyroid cancer, Graves' disease and concomitant parathyroid adenoma [20, 21]. However, it is more likely that in the future, the MIVAT technique of total thyroidectomy will be used with increasing frequency worldwide for the treatment of both benign and malignant thyroid diseases in patients with small goiters.

Presented in part to the $18^{\text {th }}$ International Congress of the European Association for Endoscopic Surgery (EAES), Geneva, Switzerland, 16-19 June 2010.

\section{References}

1. Hannan SA. The magnificent seven: a history of modern thyroid surgery. Int I Surg 2006; 4: 187-91.

2. Becker AM, Gourin CG. New technologies in thyroid surgery. Surg Oncol Clin North Am 2008; 17: 233-48.

3. Miccoli P, Berti P, Conte M, et al. Minimally invasive surgery for thyroid small nodules: preliminary report. J Endocrinol Invest 1999; 22: 849-51. 
4. Miccoli P, Berti P, Raffaelli M et al. Comparison between minimally invasive video-assisted thyroidectomy and conventional thyroidectomy: a prospective randomized study. Surgery 2001; 130: 1039-43.

5. Miccoli P, Berti P, Frustaci G, et al. Video-assisted thyroidectomy: indications and results. Langenbecks Arch Surg 2006; 391: 68-71.

6. Miccoli P, Minuto MN, Ugolini C, et al. Minimally invasive videoassisted thyroidectomy for benign thyroid disease: an evidencebased review. World J Surg 2008; 32: 1333-40.

7. Miccoli P, Pinchera A, Materazzi G, et al. Surgical treatment of low- and intermediate-risk papillary thyroid cancer with minimally invasive video-assisted thyroidectomy. J Clin Endocrinol Metab 2009; 94: 1618-22.

8. Miccoli P, Bellantone R, Mourad M, et al. Minimally invasive video-assisted thyroidectomy: multiinstitutional experience. World J Surg 2002; 26: 972-5.

9. Dzielicki J, Korlacki W, Kołodziej A, et al. Minimally invasive thyroid surgery. Pol Przegl Chir 2004; 76: 578-86.

10. Minuto MN, Berti P, Miccoli M, et al. Minimally invasive videoassisted thyroidectomy: an analysis of results and a revision of indications. Surg Endosc 2012; 26: 818-22.

11. Miccoli P, Materazzi G, Berti P. New technologies in surgery: diagnosis and treatment of complication of MIVAT (minimally Invasive Video-assisted Thyroidectomy). Pol Przegl Chir 2008; 80: 435-40.

12. Miccoli P, Berti P, Raffaelli M, et al. Impact of harmonic scalpel on operative time during video-assisted thyroidectomy. Surg Endosc 2002; 16: 663-6.

13. Barczyński M, Konturek A, Cichoń S. Minimally invasive videoassisted thyreoidectomy (MIVAT) with and without use of harmonic scalpel - a randomized study. Langenbecks Arch Surg 2008; 393: 647-54.

14. Barczyński M, Konturek A, Stopa M, et al. Total thyroidectomy for benign thyroid disease: is it really worthwhile? Ann Surg 2011; 254: 724-30.

15. Barczyński M, Konturek A, Gołkowski F, et al. Five-year followup of a randomized clinical trial of unilateral thyroid lobectomy with or without postoperative levothyroxine treatment. World J Surg 2010; 34: 1232-8.

16. Radford PD, Ferguson MS, Magill JC, et al. Meta-analysis of minimally invasive video-assisted thyroidectomy. Laryngoscope. 2011; 121: 1675-81.

17. Miccoli P, Berti P, Raffaelli M, et al. Comparison between mini mally invasive video-assisted thyroidectomy and conventional thyroidectomy: a prospective randomized study. Surgery 2001; 130: 1039-43.

18. Sahm M, Schwarz B, Schmidt S, et al. Long-term cosmetic results after minimally invasive video-assisted thyroidectomy. Surg Endosc 2011; 25: 3202-8.

19. Ujiki MB, Sturgeon C, Denham D, et al. Minimally invasive videoassisted thyroidectomy for follicular neoplasm: is there an advantage over conventional thyroidectomy? Ann Surg Oncol 2006; 13: 182-6.

20. Alesina PF, Singaporewalla RM, Eckstein A, et al. Is minimally invasive, video-assisted thyroidectomy feasible in Graves' disease? Surgery 2011; 149: 556-60.
21. Barczyński M, Cichoń S, Konturek A, et al. Comparison of two techniques of minimally invasive parathyroidectomy: videoassisted (MIVAP) and open (OMIP). Pol Przegl Chir 2007; 79: 1264-80. 